\title{
HIGH-CONTRAST POLARIZATION SPECTROSCOPY OF PHOTOCHEMICALLY BURNED SPECTRAL HOLES IN AMORPHOUS SOLIDS: POTENTIAL FOR FAST OPTICAL STORAGE
}

\author{
Bernhard DICK \\ Max-Planck-Institut für Biophysikalische Chemie, Abteilung Laserphysik, \\ Am Fassberg, D-3400 Göttingen, Federal Republic of Germany
}

Received 12 October 1987

\begin{abstract}
Polarization spectroscopy is used to detect persistent spectral holes photochemically burned into the electronic absorption band of a guest molecule in an amorphous host. Tetraphenylporphin doped into a polymethylmethacrylate (PMMA) matrix is studied as an example. The polarization technique improves the contrast ratio (increase of intensity at the hole over background) by a factor of more than 200. Applications to fast readout of optical memories and line-narrowing spectroscopy are discussed.
\end{abstract}

\section{Introduction}

Optical hole-burning is a process by which the homogeneous linewidth of a molecular transition within an inhomogeneously broadened absorption band can be obtained [ 1,2$]$. A narrow-band light source, usually a laser, excites a small fraction of molecules within the inhomogeneous ensemble, namely those that have their homogeneous absorption line in resonance with this laser. When these molecules do not return to the ground state, either due to a photochemical reaction or due to a long-lived bottleneck state [3-5], the absorption spectrum of the sample displays a narrow hole centered at the frequency of the hole-burning laser.

The spectrum of those molecules that have been removed from the inhomogeneous ensemble via holeburning may be obtained by measuring the absorption spectrum of the sample before and after holeburning, and calculating the difference. Since this is the small difference of two large quantities, very high accuracy is required in the measurement of the two absorption spectra to obtain a modest signal-to-noise ratio for the line-narrowed difference spectrum. Clearly it would be of great advantage to have a background-free method that directly measures the difference spectrum.

The hole-burning process selects not only mole- cules with a particular transition frequency, but also with a particular orientation of the transition dipole moment. Hence a hole is produced not only in the spectral inhomogeneous distribution function, but also in the orientational distribution function. As a result, an isotropic sample will become anisotropic for light with frequency close to the spectral hole. A detection technique that specifically responds to this anisotropy but gives no signal with an isotropic sample will hence provide a background-free method to detect spectral holes.

One such method, developed mainly by Wild and co-workers [6,7], makes use of an anisotropy in the form of a volume grating produced through holeburning with two overlapping coherent beams. For the detection of the holes, one of these beams is blocked and the other attenuated and used as a probe beam. When a hole is to be measured at a frequency different from the hole-burning frequency, the angle of incidence of the probe beam must be chosen according to the Bragg condition. Hence this method is not convenient for the study of satellite holes.

An alternative method is to use polarization spectroscopy. This technique is usually applied to gascous samples with transient holes produced through saturation $[8,9]$. However, applications to crystalline samples have also been reported $[10,11]$. In the present article we explore the applicability of this 
technique to the study of persistent spectral holes in amorphous solids. As an example we have chosen tetraphenylporphin doped into a polymethylmethacrylate (PMMA) matrix.

\section{Theory}

We consider a hole-burning experiment in the spectral region of an inhomogeneously broadened absorption band corresponding to a single molecular transition. A particular molecule within the inhomogeneous ensemble is characterized by the center frequency $\omega_{0}$ of its homogeneous absorption line, and its orientation with respect to the laboratory frame, measured by the set of Euler angles $\Omega=\{\alpha, \beta, \gamma\}$. The resonant contribution of the molecular transition under consideration to the polarizability can be written in the form:

$\boldsymbol{\alpha}\left(\Omega, \omega-\omega_{0}\right)=\alpha\left(\omega-\omega_{0}\right) \boldsymbol{e}_{\mu} \otimes \boldsymbol{e}_{\mu}$,

where $e_{\mu}$ is a unit vector in the direction of the molecular transition moment, and the symbol $\otimes$ denotes a tensorial product. The complex line-shape function $\alpha\left(\omega-\omega_{0}\right)$ has a contribution from the zerophonon transition and from the phonon sideband. The former contribution is

$\alpha_{z}\left(\omega-\omega_{0}\right)=\frac{\mu^{2} f}{\hbar\left(\omega-\omega_{0}-\mathrm{i} \Gamma\right)}$.

In this expression $\mu$ is the transition dipole moment, $\Gamma$ is half the homogeneous linewidth, and $f$ is the Debye-Waller factor. The absorption cross section $\sigma$ is related to the polarizability $\boldsymbol{a}$ through

$\sigma\left(\Omega, \omega-\omega_{0}\right)=\frac{4 \pi \omega}{c n} \operatorname{Im}\left(\alpha\left(\Omega, \omega-\omega_{0}\right)\right)$,

where $c$ is the speed of light and $n$ the refractive index of the sample. The inhomogeneous distribution is modelled by a distribution function $N\left(\Omega, \omega_{0}\right)$ giving the density of molecules with orientation $\Omega$ and center frequency $\omega_{0}$. The distribution function before hole-burning, $N_{0}\left(\omega_{0}\right)$, is assumed to be isotropic. Photochemical hole-burning with light of frequency $\omega_{\mathrm{B}}$, polarization $e_{\mathrm{B}}$, and fluence $F$ will produce a change in the distribution function of

$$
\begin{aligned}
& \Delta N\left(\Omega, \omega_{0}\right)=N_{0}\left(\omega_{0}\right) \\
& \quad \times\left\{\exp \left[-\Phi F e_{\mathrm{B}} \sigma\left(\Omega, \omega_{\mathrm{B}}-\omega_{0}\right) e_{\mathrm{B}}\right]-1\right\},
\end{aligned}
$$

where $\Phi$ is the quantum yield of photochemical reaction. The change in the susceptibility of the sample due to hole-burning is

$\Delta \chi(\omega)=\int \frac{\mathrm{d} \Omega}{8 \pi^{2}} \int \mathrm{d} \omega_{0} \Delta N\left(\Omega, \omega_{0}\right) \alpha\left(\Omega, \omega-\omega_{0}\right)$.

This change in susceptibility will alter the propagation properties of the sample for a probe-light beam. For the isotropic sample before hole-burning the electric field of a probe beam with polarization direction $e_{\mathrm{P}}$ propagating in the $e_{z}$ direction can be written

$$
\begin{gathered}
E=e_{\mathrm{P}} E_{0} \exp [-\mathrm{i}(\omega t-K z)], \\
K=\frac{\omega}{c} \sqrt{1+4 \pi \chi_{0}},
\end{gathered}
$$

where $\chi_{0}$ is the susceptibility of the sample before hole-burning. Due to the imaginary part of $\chi_{0}$ the length of the wave vector $K$ is complex, $K=k+\mathrm{i} a$, where $k=\omega n / c$ is the length of the usual wave vector, and $a$ is half the linear absorption coefficient of the sample. A small change $\Delta \chi$ in the susceptibility will result in a small change $\Delta E$ in the probe field amplitude. The solution of Maxwell's equation by perturbation theory yields for $\Delta E$ :

$\Delta E=-\frac{2 \pi i \omega^{2} L}{c^{2} K} \Delta \chi e_{\mathrm{P}} E_{0}$,

where $L$ is the sample thickness. The intensity $I_{\mathrm{A}}$ measured through an analyzing polarizer with polarization direction $e_{\mathrm{A}}$ is

$I_{\mathrm{A}}=\left|e_{\mathrm{A}} e_{\mathrm{P}}-\frac{2 \pi i \omega^{2} L}{c^{2} K} e_{\mathrm{A}} \Delta \chi e_{\mathrm{P}}\right|^{2} I_{\mathrm{P}} \operatorname{cxp}(-2 a L)$,

where $I_{\mathrm{P}}$ is the intensity of the probe beam before it enters the sample. Hence, a background-free holeburning spectrum can be obtained when $e_{\mathrm{A}}$ is perpendicular to $e_{\mathrm{P}}$. The condition is that neither $e_{\mathrm{P}}$ nor $e_{\mathrm{A}}$ must be an eigenvector of $\Delta \chi$. Since $\Delta \chi$ is axially symmetric around the polarization vector $e_{B}$ of the light used for hole-burning, this condition is fulfilled when $e_{\mathrm{P}}$ is neither parallel nor perpendicular to $e_{\mathrm{B}}$. For small photochemical turnover eq. (3) can be ex- 
panded and only the term linear in $F$ retained. In this case eq. (4) leads to

$$
e_{\mathrm{A}} \Delta \chi e_{\mathrm{P}}=-\Phi F G H\left(\omega, \omega_{\mathrm{B}}\right) \text {, }
$$

with

$$
\begin{aligned}
& G=\int \frac{\mathrm{d} \Omega}{8 \pi^{2}}\left(e_{\mu} e_{\mathrm{A}}\right)\left(e_{\mu} e_{\mathrm{P}}\right)\left(e_{\mu} e_{\mathrm{B}}\right)^{2} \\
& \quad=\frac{1}{3}\left(e_{\mathrm{A}} e_{\mathrm{P}}\right)+\frac{2}{15}\left(e_{\mathrm{A}} e_{\mathrm{B}}\right)\left(e_{\mathrm{B}} e_{\mathrm{P}}\right), \\
& H\left(\omega, \omega_{\mathrm{B}}\right)=\int \mathrm{d} \omega_{0} N_{0}\left(\omega_{0}\right) \sigma\left(\omega_{\mathrm{B}}-\omega_{0}\right) \alpha\left(\omega-\omega_{0}\right) .
\end{aligned}
$$

Hence, with $e_{\mathrm{A}}$ perpendicular to $e_{\mathrm{P}}$, the lineshape of the hole is $\left|H\left(\omega, \omega_{\mathrm{B}}\right)\right|^{2}$ and its amplitude increases with $F^{2}$. The maximum signal is expected when $e_{\mathrm{P}}$ is inclined by $\pi / 4$ with respect to $e_{\mathrm{B}}$. The corresponding lineshape measured by the usual technique without polarizers is proportional to $\operatorname{Im}\left(H\left(\omega, \omega_{\mathrm{B}}\right)\right)$, and its amplitude increases linearly with the fluence $F$.

\section{Experimental}

Meso-tetraphenylporphin was obtained from EGAChemie and used without further purification. Stock solutions of tetraphenylporphin in chloroform with concentrations between $10^{-3}$ and $10^{-4} \mathrm{M}$ were prepared containing $2.5 \mathrm{~g}$ polymethylmethacrylate
(PMMA, Elvacit 2000 from DuPont) in $10 \mathrm{ml}$. Films were produced by casting $\approx 1 \mathrm{ml}$ solution onto a 25 $\mathrm{mm}$ diameter sapphire substrate and evaporation of the solvent. Typical films had a thickness of 200-350 $\mu \mathrm{m}$ and an optical density between 0.4 and 1.1 in the wavelength range of the electronic origin of tetraphenylporphin $(6455 \AA)$.

The experimental apparatus is shown schematically in fig. 1 . The sapphire substrate covered with the sample film was mounted on the cold finger of a refrigerator cryostat (Leybold ROK 10-300) and cooled to $10 \mathrm{~K}$ A pulsed dye laser (Lambda Physik FL 2002) pumped by a XeCl excimer laser (Lambda Physik EMG 101) was used for burning and detection of the spectral holes. The dye laser output was partially polarized ( $\approx 20: 1$ ). Part of the dye laser beam was split off and used as a reference beam to monitor the fluctuations in the dye laser output. The main beam was directed through the cryostat and the sample placed between two Glan prism polarizers. A double Fresnel rhombus acting as an achromatic halfwave plate was used to rotate the plane of polarization to achieve maximum transmission through the polarizer P1. Each beam was detected by a 1 P28 photomultiplier mounted on the end of a $30 \mathrm{~cm}$ long tube with a $1 \mathrm{~mm}$ pinhole at the other end. Color filters with a cutoff at $5300 \AA$ served to reduce the noise from residual room light.

Sapphire is a birefringent material, and our sub-

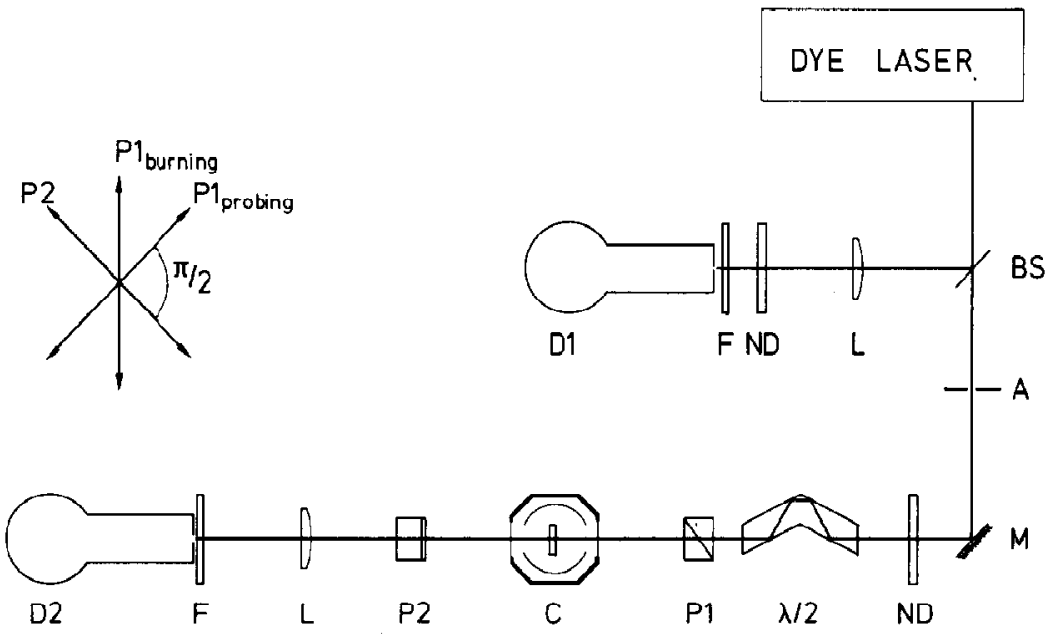

Fig. 1. Experimental apparatus. A, aperture; BS, beam splitter; D, detector (1P28 photomultiplier); F, color filter (Schott GG 530,3 $\mathrm{mm}$ ); $C$, cryostat; $L$, lens; $M$, mirror, $N D$, neutral density filter; $P$, Glan polarizer. 
strates had random orientation of the optical axis. Hence the polarizer P1 must be oriented in such a way that the beam passes the sapphire as a purely ordinary or purely extraordinary ray. This orientation was found by successive rotation of both polarizers to achieve minimum transmission. In this way an extinction of $3.5 \times 10^{-5}$ before hole-burning could be attained. For hole-burning the polarizer P1 was turned $45^{\circ}$ and the neutral density filters removed. The laser pulses had an average fluence of $35 \mu \mathrm{J} / \mathrm{cm}^{2}$ illuminating an area of $\approx 3 \mathrm{~mm}$ diameter determined by the size of the aperture A. For probing the polarizer $P 1$ was rotated back to its original position, and the laser attenuated by a factor of approximately $10^{4}$.

\section{Results and discussion}

\subsection{Comparison of crossed-polarizer and parallel- polarizer configurations}

Fig. 2 shows the transmission spectrum of a hole burned into the electronic origin band of tetraphen-

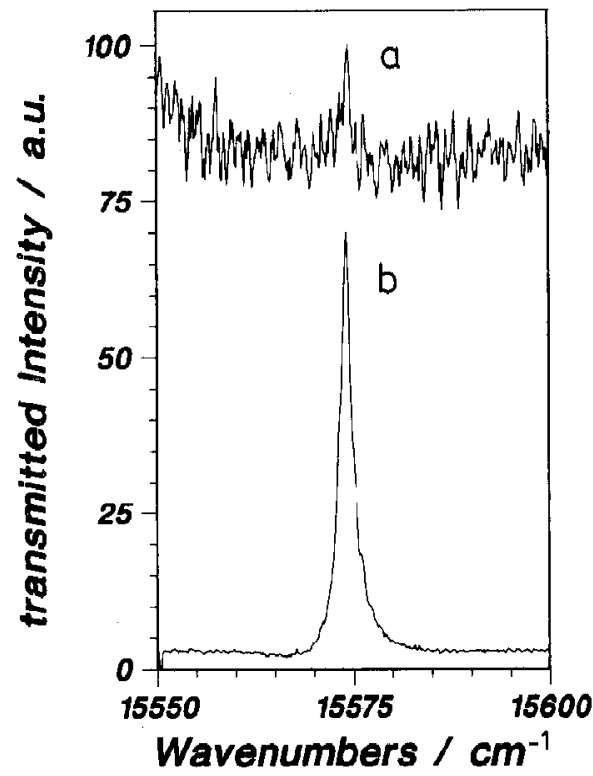

Fig. 2. Lineshape of a hole burned into the electronic origin band of tetraphenylporphin in a PMMA matrix. The intensity of the transmitted light is measured in arbitrary units. (a) Polarizers $P 1$ and $P 2$ parallel, (b) polarizers $P 1$ and $P 2$ perpendicular. ylporphin in PMMA matrix at $10 \mathrm{~K}$. Curve (a) was obtained with $e_{\mathrm{A}}(\mathrm{P} 2)$ parallel to $e_{\mathrm{P}}$ (P1), where curve (b) was obtained with crossed polarizers. The probe laser had shot-to-shot fluctuations of approximately $10 \%$, which is of the same order of magnitude as the increase in transmission at the center of the hole. (The sample had an optical density of 0.75 , and the change in optical density due to hole-burning was approximately 0.04 .) Hence curve (a) is very noisy; the hole is hardly visible and might even be overlooked. On the other hand, the spectrum obtained with crossed polarizers is well resolved and practically unaffected by noise. We can define a contrast ratio as the ratio of the increase in transmitted light intensity at the center of the hole to the background intensity. This ratio is $\approx 0.13$ for curve (a) and $\approx 27$ for curve $(b)$. Hence the use of the crossedpolarizer configuration increased the contrast ratio by a factor of more than 200 . The background in curve (b) is largely due to residual strain birefringence in the cryostat windows and the sapphire substrate. These contributions could be reduced through careful selection of strain-free materials, or compensated with a Soleil-Babinet compensator. Thus an even greater contrast ratio should be possible. In the vicinity of a zero-phonon hole the lower limit for the background level will be given by the anisotropy produced through hole-burning into the phonon sideband.

The noise level in the measurement of the probe light intensity could be greatly reduced through the use of $\mathrm{cw}$ light sources and detection with photoncounting or lock-in techniques. However, this would improve both curves (a) and (b) to the same extent. The results of three hole-burning series performed with increasing laser fluence at different wavelengths within the electronic origin band of tetraphenylporphin in a PMMA matrix are shown in fig. 3. The symbols represent the experimental data, whereas the full lines indicate the quadratic dependence predicted by the theory for small photochemical turnover. The ideal quadratic behavior is well observed up to approximately 200 accumulated laser shots corresponding to a fluence of $\approx 7 \mathrm{~mJ} / \mathrm{cm}^{2}$. For higher fluences saturation sets in. In the spectra this saturation is apparent from the growth of the phononsideband hole relative to the zero-phonon hole. It should be mentioned that at $10 \mathrm{~K}$, the lowest tem- 


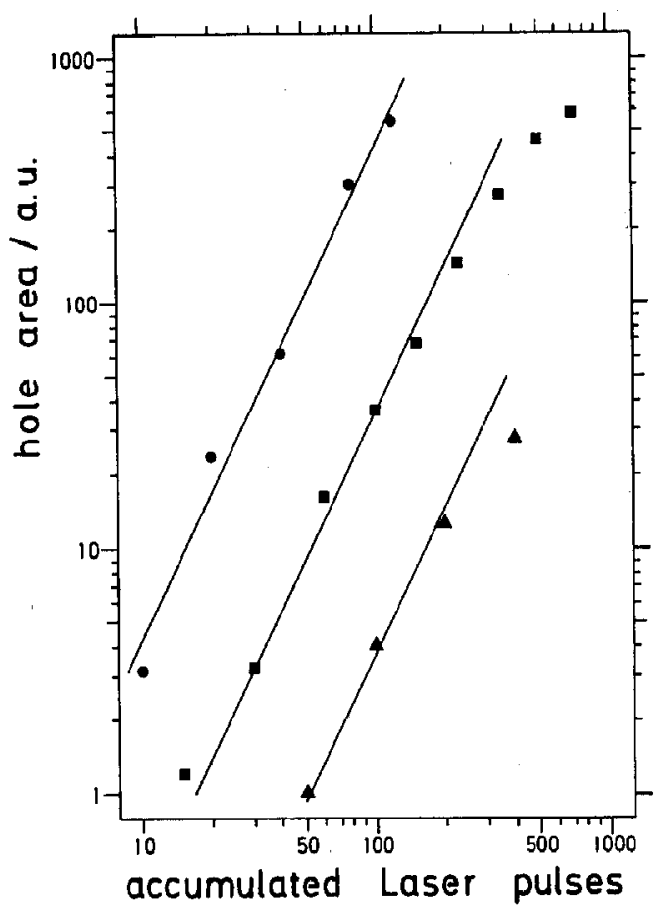

Fig. 3. Growth of the hole area (in arbitrary units) with laser fluence, measured using the crossed-polarizer technique. The various symbols correspond to experiments at different wavelengths and with different samples. The full lines indicate the theoretical quadratic relation

perature accessible with our present apparatus, the homogeneous linewidth of the electronic origin of tetraphenylporphin is still dominated by pure dephasing. At lower temperatures, and with narrow-band lasers, saturation should be reached with much smaller fluences.

\subsection{Optical storage application}

The detection of spectral holes through crossed polarizers could be useful for the readout of optical data storage devices. As a simulation of such an optical memory a bit pattern (10111) was burned into the rising edge of the electronic origin band of tetraphenylporphin in PMMA. Fig. 4 shows the transmission spectrum of this sample measured with parallel polarizers (upper part) and between crossed polarizers (lower part). With detection through parallel polarizers, measurement of the transmitted light intensity at a single frequency is not sufficient to distinguish a " 1 " (hole) from a " 0 ". The reason is that

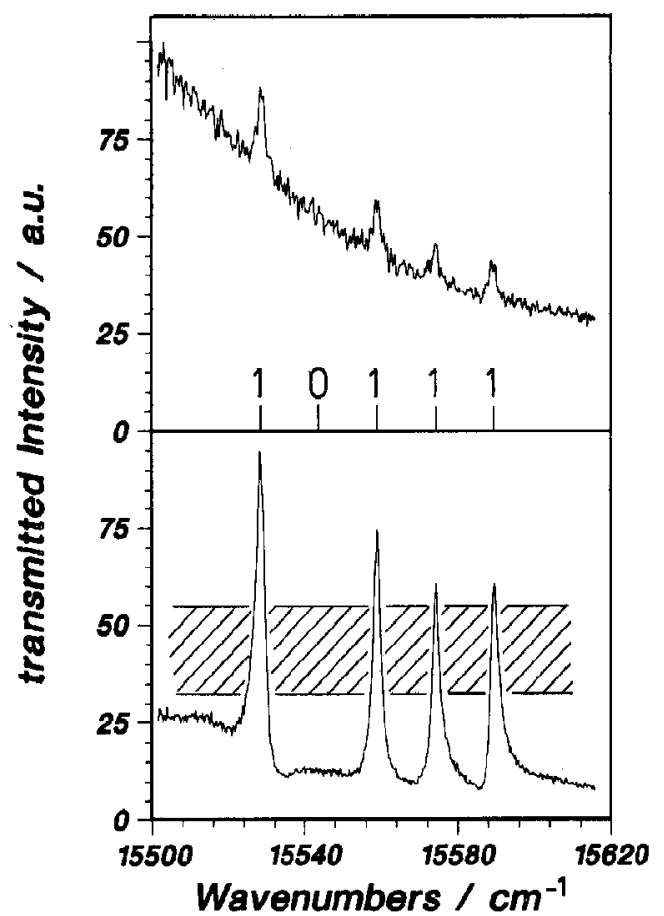

Fig. 4. Optical storage application of the crossed-polarizer technique. A bit pattern (10111) was burned into the long wavelength side of the electronic origin band of tetraphenylporphin in a PMMA matrix. Upper part: transmission spectrum of the sample measured with parallel polarizers, lower part: transmission spectrum measured with crossed polarizers.

a hole leads only to a small increase of transmitted light intensity which is much smaller than the intensity variation across the absorption band. Hence no threshold light level for discrimination exists, and at least two measurements at slightly different frequencies are needed to determine the value of a particular bit. When this is done with modulation techniques, a minimum time of the order of a few cycles of the modulation are required. However, when the readout is performed through crossed polarizers, a "1" corresponds to a high light level, whereas a " 0 " is essentially dark. A threshold light level could be easily defined within the region indicated through shading in the lower part of fig. 4 . Hence readout of a particular bit in the memory requires only a single light pulse which could be as short as a few picoseconds. Of course, the spectral width of these read pulses would put an upper limit to the spectral density of holes, and hence to the storage density of such a device. The holes in fig. 4 have a width of $2.5 \mathrm{~cm}^{-1}$ 
determined by the bandwidth of the dye laser used for hole-burning and probing. This spectral width would be closely matched by the spectral width of a Gaussian light pulse of 6 ps duration. A laser with a lower bandwidth, e.g. $0.1 \mathrm{~cm}^{-1}$, would allow storage of more than $10^{4}$ bits within the inhomogeneous band of the electronic origin of tetraphenylporphin which could each be read in 150 ps. In fig. 4, the holes have been burned up to the beginning of saturation in order to render them more visible in the parallel-polarizer configuration. This led to a contribution to the background from phonon sidebands, which would be considerably reduced if lower fluences were used for hole-burning.

\subsection{Application to line-narrowing spectroscopy}

The photochemical hole-burning process removes molecules with a molecular transition in resonance with the excitation laser from the inhomogeneous ensemble. So far we have only considered the change in susceptibility that arises from the contribution of this molecular transition to the polarizability of the removed molecules. However, the polarizabilities of the removed molecules have resonances for all molecular transitions, and hence a satellite hole should appear for each molecular transition with sufficient oscillator strength. Whereas the linewidth of the resonant holes is determined by the inverse $T_{2}$ relaxation time of the molecular level, the linewidth of a non-resonant satellite hole contains an additional contribution determined by the degree of correlation between the inhomogeneous distributions of the burned and the probed molecular transition. For vibrational levels of the same electronic state the inhomogeneous distributions are usually strongly correlated and hence narrow satellite holes are expected. In such cases a measurement of the satellite holes will yield a line-narrowed spectrum of a small subset of the guest molecules in the amorphous surroundings. When hole-burning is performed in the usual way with parallel polarization of $e_{\mathrm{P}}$ and $\boldsymbol{e}_{\mathrm{A}}$, this line-narrowed spectrum will be the difference of the absorption spectra taken before and after hole-burning. This change in absorption is very small, usually less than $1 \%$, and hence the measurement of a linenarrowed spectrum suffers from the usual problems associated with the measurement of the small dif-

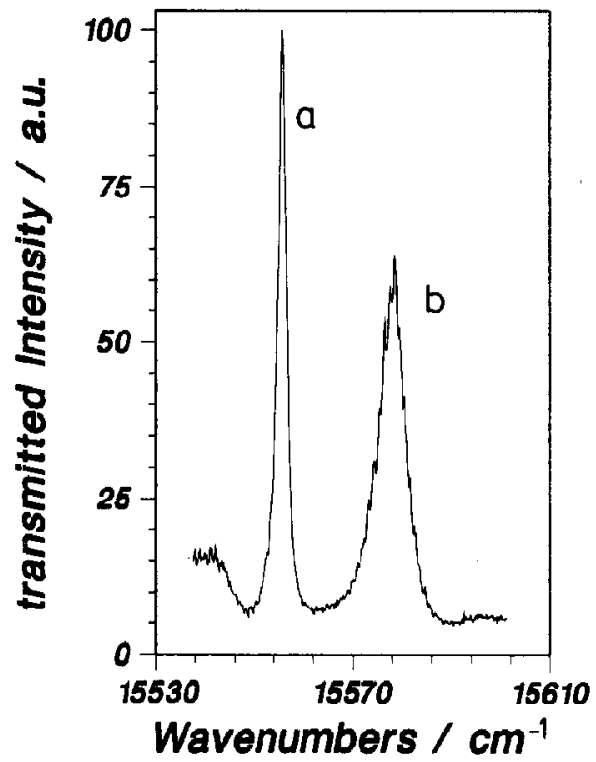

Fig. 5. Lineshape of a resonant hole (a) and a satellite hole (b) both observed within the inhomogeneous bandwidth of the electronic origin of tetraphenylporphin in a PMMA matrix. The resonant hole (a) was burned at $15556 \mathrm{~cm}^{-1}$ into the electronic origin band, the satellite hole was burned at $17085 \mathrm{~cm}^{-1}$ into a vibronic band and is observed at $15578 \mathrm{~cm}^{-1}$.

ference of large quantities. In the crossed-polarizer configuration, however, this line-narrowed spectrum can be obtained directly in a single scan. Fig. 5 shows a scan displaying two holes in the region of the electronic origin band of tetraphenylporphin in PMMA matrix. Curve (b) is a non-resonant satellite hole. It was burned at $17085 \mathrm{~cm}^{-1}$ into a vibrational level of the same electronic state, and it is found here with a peak at $15578 \mathrm{~cm}^{-1}$. The shift between the holeburning frequency and the center frequency of the non-resonant hole is $1507 \mathrm{~cm}^{-1}$ in good agreement with the frequency shift of $1505 \mathrm{~cm}^{-1}$ of an intense vibronic line found in the fluorescence excitation spectrum of tetraphenylporphin in a Shpolskii matrix [12]. Curve (a) in fig. 5 is a resonant hole burned at $15556 \mathrm{~cm}^{-1}$ for comparison. The width of the nonresonant hole is only $6 \mathrm{~cm}^{-1}$ compared to the 300 $\mathrm{cm}^{-1}$ wide inhomogeneous bandwidth, demonstrating the line-narrowing effect. The resonant hole is even narrower with a width of $2.5 \mathrm{~cm}^{-1}$, which is mainly due to the linewidth of our laser. The observed difference in linewidth for the two holes could reflect a small deviation from perfect correlation of the two levels, but is more probably due to the shorter 
lifetime of the vibronic state. If we interpret the resonant hole as the response function of our apparatus, the deconvoluted linewidth of the non-resonant hole is $5.5 \mathrm{~cm}^{-1}$ corresponding to a lower limit to the lifetime of 1 ps. A discussion of line-narrowed spectra together with a more comprehensive theoretical treatment of the satellite hole phenomenon will be given elsewhere.

\section{Conclusions}

The sensitivity of the detection of optical holes burned into inhomogeneously broadened absorption bands can be greatly increased through employment of polarization spectroscopy. In the example presented here - tetraphenylporphin in a PMMA matrix - persistent photochemical holes have been studied, but the method should also be applicable to photophysical or transient holes.

Much higher sensitivity than that demonstrated here should be possible with some straightforward improvements of the apparatus, e.g. through:

(i) using a $\mathrm{cw}$ light source for scanning the holes;

(ii) using photon-counting techniques or optical multichannel detection;

(iii) selecting strain-free window materials or compensation of strain birefringence with a SoleilBabinet compensator;

(iv) placing the sample directly into liquid helium, thus avoiding the sapphire substrate.

\section{Acknowledgement}

We thank Dr. N. Ernsting for valuable discussions, and Professor F.P. Schäfer for support. This work was also supported by the Deutsche Forschungsgemeinschaft under SFB 93 (Photochemie mit Lasern).

\section{References}

[1] L.A. Rebane, A.A. Gorokhovskii and J.V. Kikas, Appl. Phys. B29 (1982) 235.

[2] J. Friedrich and D. Haarer, Angew. Chem. 96 (1984) 96; Angew. Chem. Intern. Ed. 23 (1984) 113.

[3] R.M. Shelby and R.M. Macfarlane, Chem. Phys. Letters 64 (1979) 545 .

[4] A.I.M. Dicker, L.W. Johnson, S. Völker and J.H. van der Waals, Chem. Phys. Letters 100 (1983) 8.

[5] B. Dick and B. Nickel, Chem. Phys. 110 (1986) 131.

[6] A.J. Meixner, A. Renn. S.E. Bucher and U.P. Wild, J. Phys. Chem. 90 (1986) 6777.

[7] R. Locher, A. Renn and U.P. Wild, Chem. Phys. Letters 138 (1987) 405 .

[8] C. Wiemann and T.W. Hänsch, Phys. Rev. Letters 36 (1976) 1170.

[9] J.J. Song, J.H. Lee and M.D. Levenson, Phys. Rev. 17 (1978) 1439.

[10] J.H. Lee, J.J. Song, M.A.F. Scarparo and M.D. Levenson, Opt. Letters 5 (1980) 196.

[11] M.D. Levenson, R.M. Macfarlane and R.M. Shelby, Phys. Rev. B22 (1980) 4915.

[12] R. Tamkivi, I. Renge and R. Avarmaa, Chem. Phys. Letters 103 (1983) 103. 